\title{
A histopathological connection between a fatal endolymphatic sac tumour and von Hippel-Lindau disease from 1960
}

\author{
J A BELLAIRS ${ }^{1,2}$, M B GLUTH ${ }^{2}$ \\ ${ }^{1}$ Pritzker School of Medicine, University of Chicago, and ${ }^{2}$ Section of Otolaryngology - Head and Neck Surgery, \\ University of Chicago Medical Center, Illinois, USA
}

\begin{abstract}
Objective: To highlight a case from 1960 connecting endolymphatic sac tumour and von Hippel-Lindau disease.

Case report: In 1960, a 24-year-old woman presented with unilateral hearing loss, pulsatile tinnitus and a mass visible on otoscopy. The patient underwent surgical biopsy, which was complicated by haemorrhage, and ultimately resulted in death. At autopsy, a destructive temporal bone neoplasm with cystic and papillary architecture was observed that had eroded into the otic capsule. Intra-abdominal lesions consistent with von Hippel-Lindau disease were also observed, and the surgeon postulated a connection between endolymphatic sac tumour and von Hippel-Lindau disease.

Method: A review of the literature was carried out using PubMed.

Conclusion: Endolymphatic sac tumours are rare neoplasms of the temporal bone that can occur sporadically or as part of von Hippel-Lindau disease. The connection between endolymphatic sac tumour and von Hippel-Lindau disease was first proposed in 1988 and formalised in 1997. We believe that this case represents the first documented connection between endolymphatic sac tumour and von Hippel-Lindau disease.
\end{abstract}

Key words: Endolymphatic Sac; Endolymphatic Hydrops; Von Hippel-Lindau Disease; Temporal Bone

\section{Introduction}

Endolymphatic sac tumours are benign, locally aggressive tumours that arise from the epithelial layer of the endolymphatic duct and sac. The endolymphatic sac is located along the posterior plate of the petrous bone, and is primarily responsible for maintaining endolymphatic fluid volume, ionic equilibrium and pressure. Endolymphatic sac tumours extend in the direction of the bony labyrinth. Affected patients frequently present with a Ménière-like constellation of unilateral sensorineural hearing loss, vertigo and tinnitus.

Endolymphatic sac tumours can arise from sporadic mutations, but they also occur as a phenotypic manifestation of von Hippel-Lindau disease. ${ }^{1}$ These tumours are also exceedingly rare, with only approximately 200 cases reported in the literature since they were first characterised in the late 1980s.

Here, we report a historical case of an endolymphatic sac tumour that came to medical attention in 1960. This patient presented to Luton and Dunstable Hospital in the UK, and was seen by Mr Adrian Griffith.

The patient underwent a surgical biopsy, but unfortunately she suffered a fatal cardiac arrest in the post-operative period. At autopsy, a cystic lesion originating in the temporal bone was observed, in addition to intra-abdominal lesions consistent with von Hippel-Lindau disease. From this examination, Dr Griffith suggested a connection between von Hippel-Lindau disease and this patient's temporal bone lesion. For further histological characterisation of this cyst, the patient's temporal bone was sent to Dr John Lindsay at the University of Chicago.
Dr Lindsay's report confirmed that the lesion was an erosive and highly vascular papillary tumour originating from the area of the endolymphatic sac. Unfortunately, Mr Griffith suffered an untimely death and never learned of this histopathological diagnosis, which ultimately confirmed his hypothesis.

We present Mr Griffith's and Dr Lindsay's reports, and provide a brief history of endolymphatic sac tumours, to demonstrate what we believe to be the first recorded connection between endolymphatic sac tumour and von Hippel-Lindau disease.

\section{Case report}

The following is an abbreviated quotation from a letter sent from Mr Griffith to Dr Lindsay dated 14th March 1961:

'This patient was first seen by Mr Capps at the Luton and Dunstable Hospital in December 1960. Aged 24, British, but of rather dark appearance. Her general configuration suggested possibly she might have a minor degree of Turner's syndrome, pigmented and short of neck, coarse skin, pronounced hairiness, etc. There was no significant family or past history. She complained of a unilateral left-sided deafness and pulsating tinnitus, and occasional headaches. There had been no ear discharge and no vertigo'.

'At examination the nose and throat were normal, but the left middle ear was filled with a dark purplish mass which was seen to pulsate. There was no hearing. Tests 
of vestibular function were not carried out. No bruit could be heard over the affected ear'.

'The appearances were very suggestive of a glomus jugulare tumour, of which Mr Capps has experience of some 35 cases... Radiography of the temporal bones was carried out and considerable erosion was seen, which was confirmed by tomography. There was a curious appearance of abnormal bone formation, which did in some ways suggest an osteogenic sarcoma. However, we made a diagnosis of a glomus jugulare tumour'.

'Our normal treatment for these is a full course of radiotherapy and in our last 30 cases this has proved extremely effective, and we have not had to resort to surgery. On this particular occasion, however, it was decided to obtain a biopsy through the mastoid, and with several pints of blood at hand a rapid mastoidectomy and clearance of the vascular tumour was carried out. The bleeding was considerable, but controlled with a pack. Hypotension... was employed throughout the operation, and the blood pressure dropped to about $50 \mathrm{~mm} \mathrm{Hg}$ at one point, but recovered to more normal levels at the end of the operation. A few hours after this she suddenly collapsed. The chest was opened, cardiac massage carried out, and the heart beat restored, but she did not recover consciousness and died some two days later. During this period the circulation was extremely poor and her temperature remained subnormal for [24] hours, and for a long

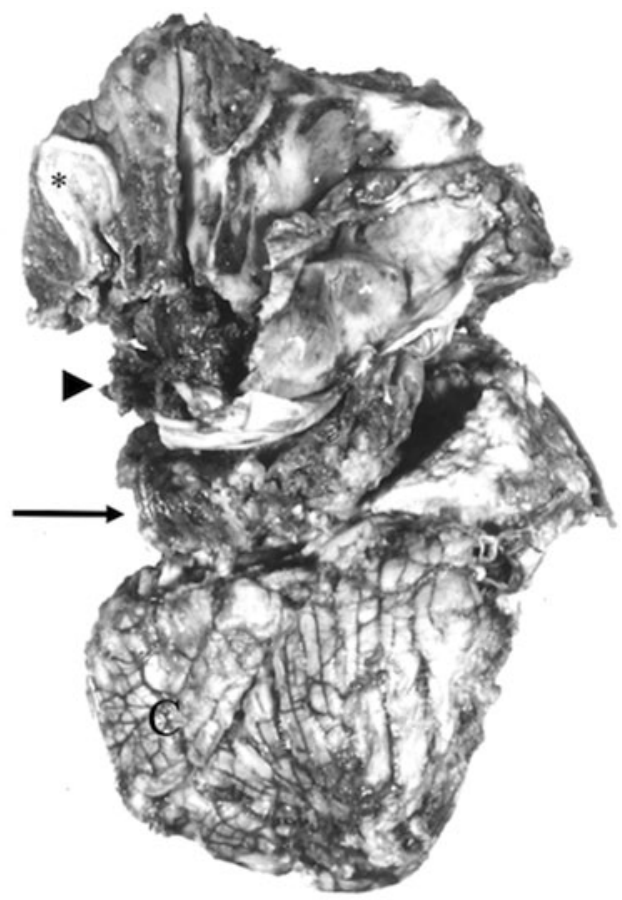

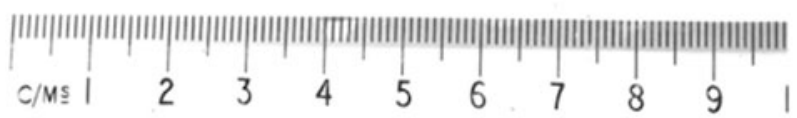

FIG. 1

Left temporal bone and cerebellum gross specimen. Pre-processing photograph of left temporal bone and cerebellum specimen submitted to Dr Lindsay. Gross examination revealed transected external auditory canal (asterisk), surgical mastoidectomy cavity filled with blood clot (arrowhead), cystic tumour (arrow) and cerebellum ('C'). period it was frankly very difficult to determine whether she was alive or not...'

'[At autopsy] the temporal bones were removed with the cerebellum, and this is the specimen which I sent to you. I have taken some photographs [(Figure 1)] and enclose these... The post mortem disclosed this large cystic tumour involving the region of the cerebellum, the mastoid and labyrinth and presenting at the tympanic membrane'.

'When the pancreas and kidneys were examined they were found to be full of cysts and a section of these shows them to be cyst-adenomatous with considerable activity, and they cannot be regarded as simple benign cysts'.

'I feel that this is not a case of a glomus jugulare but a form of von Hippel-Lindau disease, in which the left temporal bone has become involved. Cysts are often found in the cerebellum, but not I think in the temporal bone. Further, it seems doubtful that the tumour could have spread from the cerebellum, through the mastoid to be present at the middle ear, and it would appear

(a)

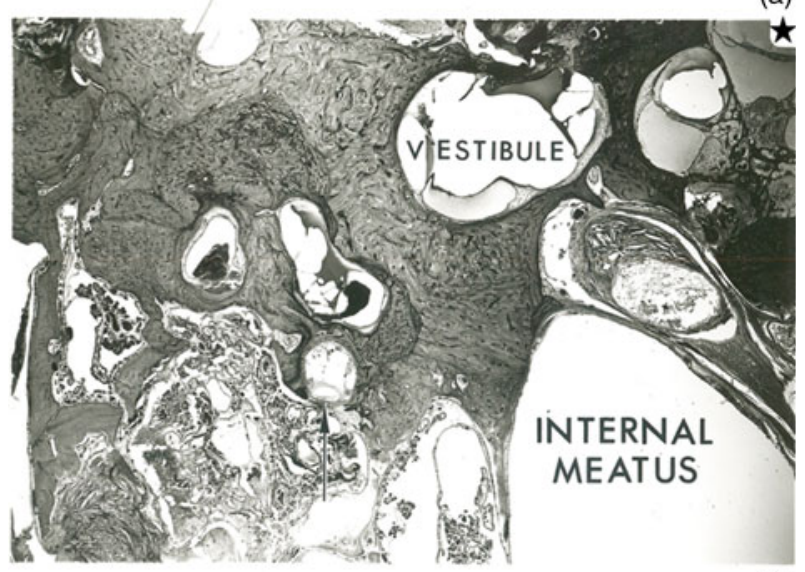

(b)

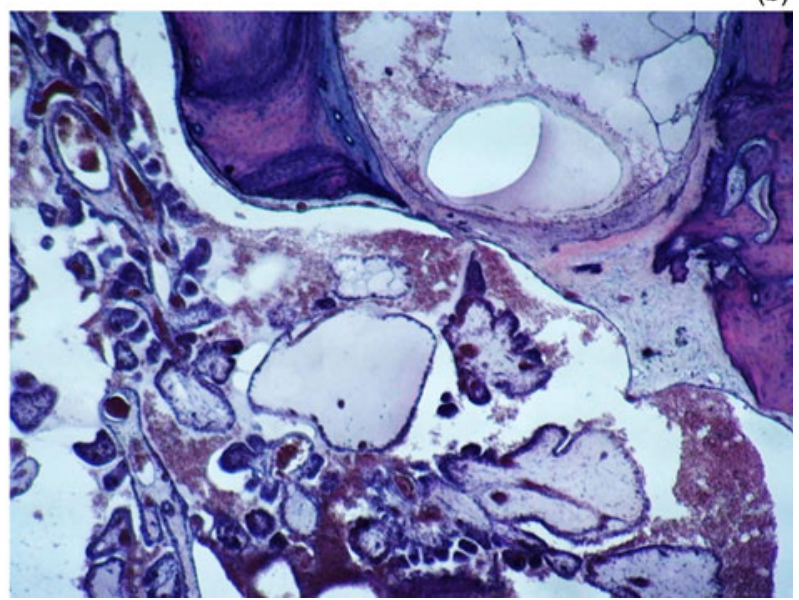

FIG. 2

Histopathological examination of left temporal bone. Histopathological specimen prepared by Dr Lindsay demonstrates cystic-papillary tumour architecture, endolymphatic hydrops and temporal bone erosion. (a) Original photomicrograph highlights the vestibule, tumour erosion up to the internal auditory meatus, endolymphatic hydrops (star) and tumour erosion into the posterior semicircular canal (arrow) $(\mathrm{H} \& \mathrm{E} ; \times 11)$. (b) Recent photomicrograph demonstrates magnified view of papillary tumour eroding into posterior semicircular canal. $(\mathrm{H} \& \mathrm{E} ; \times 40)$ 
more likely that this was a rest or development of a vascular tumour in the temporal bone itself growing laterally into the middle ear and medially into the cerebellum. I am hopeful that the sections of this extremely interesting case will clear up this question'.

The following is an excerpt from Dr John Lindsay's histopathological report:

'Otic Capsule: The posterior surface of the pyramid including the walls of the internal meatus, but mainly posterior to it is occupied by tumour. The tumour consists of deep staining villi with relatively little sub-epithelial structure, highly vascular lying in spaces which are partly clear and non-cellular, partly filled with free red blood cells. The posterior layer of the capsule shows evidence of absorption and replacement of bone - partly web like, highly cellular and partly lamellar [(Figure 2)]. In places the endochondral layer has been absorbed and fistulas have occurred. The fistulas are mainly into the common crus and posterior canal'.

The diagnosis was reported by Dr Lindsay as: ' 1 . Tumor, Benign - von Hippel-Lindau disease. 2. Hydrops secondary’.

\section{Discussion}

The first report of endolymphatic sac tumour was made by Hassard et al., in 1984, when a tumour originating in the endolymphatic sac was discovered during an endolymphatic sac decompression for Ménière's disease. ${ }^{2}$ The initial pathological diagnosis was a choroid plexus papilloma; however, surgical exploration revealed that the tumour was entirely extradural. Subsequent review led to reclassification of the tumour as an adenoma of the endolymphatic sac. ${ }^{2}$

- Endolymphatic sac tumours are rare, benign, locally aggressive lesions, derived from endolymphatic sac and duct epithelia

- These tumours often present with a Ménière-like constellation of sensorineural hearing loss, tinnitus and vertigo

- The tumours were first reported in the literature in 1984, and further characterised in $\mathbf{1 9 8 8}$

- Tumours can occur sporadically or with von Hippel-Lindau disease, with the latter occurring in younger, often female patients

- The endolymphatic sac tumour and von Hippel-Lindau disease connection was first proposed in 1988, and formalised in $\mathbf{1 9 9 7}$

- This case documents a proposal of this connection from 1960, nearly 30 years before it was reported in the literature

In 1988, Gaffey et al. proposed that these tumours be classified as 'aggressive papillary middle ear tumours'. ${ }^{3}$ That retrospective series highlighted nine cases of locally invasive temporal bone tumours with papillary architecture. ${ }^{3}$ In 1989 , Heffner published a seminal clinicopathological review of 20 cases that he described as 'low grade adenocarcinoma of probable endolymphatic sac origin'. ${ }^{4}$ Pathologically, the tumours were noted to have uniform papillary-cystic architecture, with infiltration of the posterior wall of the petrous bone. In that series, the ages of the patients ranged from 15 to 71 years, with a mean age of 41 years. Clinically, sensorineural hearing loss was the primary presenting symptom, with an average duration of 9.3 years. ${ }^{4}$ In 1993, Li et al. proposed reclassifying all adenomatous tumours of the endolymphatic sac as endolymphatic sac tumours, to better delineate them from other neoplasms affecting the temporal bone. ${ }^{5}$

Endolymphatic sac tumours occur sporadically or as a part of von Hippel-Lindau disease. von Hippel-Lindau disease is an autosomal dominant disorder, with a prevalence of 1 in 36000 live births. The disease presents with cerebellar haemangioblastomas, retinal angiomas, renal cysts, renal carcinoma, pheochromocytoma, pancreatic cysts, epididymal cysts and endolymphatic sac tumour. ${ }^{6}$

The connection between von Hippel-Lindau disease and endolymphatic sac tumour was first proposed by Eby et al., in 1988, who described a von Hippel-Lindau disease patient with bilateral lesions and a strong family history. ${ }^{7}$ Endolymphatic sac tumour was formally recognised as part of von Hippel-Lindau disease in 1997. The prevalence of von Hippel-Lindau disease patients that develop endolymphatic sac tumour is now estimated to be between 3 and 16 per cent. ${ }^{8-10}$ Compared to sporadic cases, endolymphatic sac tumour in von Hippel-Lindau disease presents at a younger age (mean, 31.3 years) and with a female preponderance $\left(2: 1\right.$ ratio). ${ }^{11}$

In this report, we present what we believe to be the first documented connection between endolymphatic sac tumour and von Hippel-Lindau disease. Clinical features of this presentation consistent with the diagnosis of endolymphatic sac tumour include unilateral hearing loss, tinnitus, and a mass involving the mastoid and the middle ear. Gross examination revealed a highly vascular, cystic and friable tumour (Figure 1), while microscopic evaluation demonstrated an infiltrating tumour with papillary and cystic architecture (Figure 2). This constellation of clinical and pathological findings is certainly deserving of the modern diagnosis of endolymphatic sac tumour. While we have no definitive proof of the diagnosis of von Hippel-Lindau disease, the patient's young age and the autopsy findings are consistent with this syndrome. The most remarkable aspect of this case, however, is Mr Griffith's postulation that von Hippel-Lindau disease and endolymphatic sac tumour are connected. This documented hypothesis comes more than 30 years before the connection was formally established. Most unfortunately, he suffered an untimely demise, before his observations could be published. ${ }^{12}$ We hope that this report highlights a brilliant insight that was truly ahead of its time.

\section{Acknowledgements}

The authors would like to acknowledge Mr Adrian Griffith for the description of the case and specimen collection, and Dr John Lindsay for temporal bone preparation and histopathological interpretation. To the best of our knowledge, this case has not previously been published elsewhere. $\mathrm{JAB}$ and MBG contributed equally to the background research and manuscript preparation. This research was supported by the National Institute on Deafness and Other Communication Disorders of the National Institutes of Health, under award number U24DC015910.

\section{References}

1 Bailey BJ, Johnson JT, Newlands SD. Head \& Neck Surgery-Otolaryngology. Philadelphia: Lippincott Williams \& Wilkins, 2006;3053 
2 Hassard AD, Boudreau SF, Cron CC. Adenoma of the endolymphatic sac. J Otolaryngol 1984;13:213-16

3 Gaffey MJ, Mills SE, Fechner RE, Intemann SR, Wick MR. Aggressive papillary middle-ear tumor. A clinicopathologic entity distinct from middle-ear adenoma. Am J Surg Pathol 1988;12:790-7

4 Heffner DK. Low-grade adenocarcinoma of probable endolymphatic sac origin. A clinicopathologic study of 20 cases. Cancer 1989;64:2292-302

5 Li JC, Brackmann DE, Lo WW, Carberry JN, House JW. Reclassification of aggressive adenomatous mastoid neoplasms as endolymphatic sac tumors. Laryngoscope 1993;103:1342-8

6 Lonser RR, Glenn GM, Walther M, Chew EY, Libutti SK, Linehan WM et al. von Hippel-Lindau disease. Lancet 2003; 361:2059-67

7 Eby TL, Makek MS, Fisch U. Adenomas of the temporal bone. Ann Otol Rhinol Laryngol 1988;97:605-12

8 Manski TJ, Heffner DK, Glenn GM, Patronas NJ, Pikus AT, Katz D et al. Endolymphatic sac tumors. A source of morbid hearing loss in von Hippel-Lindau disease. JAMA 1997;277: 1461-6

9 Bausch B, Wellner U, Peyre M, Boedeker CC, Hes FJ, Anglani $\mathrm{M}$ et al. Characterization of endolymphatic sac tumors and von Hippel-Lindau disease in the International Endolymphatic Sac Tumor Registry. Head Neck 2016;38:E673-9
10 Choo D, Shotland L, Mastroianni M, Glenn G, van Waes C, Linehan WM et al. Endolymphatic sac tumors in von HippelLindau disease. J Neurosurg 2004;100:480-7

11 Bambakidis NC, Megerian CA, Ratcheson RA. Differential grading of endolymphatic sac tumor extension by virtue of von Hippel-Lindau disease status. Otol Neurotol 2004;25: $773-81$

12 Obituary: A. N. GRIFFITH, M.B., B.Chir., F.R.C.S., D.L.O. $\mathrm{Br}$ Med J 1961;2:1296

Address for correspondence:

Dr Michael B Gluth,

Section of Otolaryngology - Head and Neck Surgery,

University of Chicago Medical Center,

5841 S. Maryland Ave, MC 1035,

Chicago, IL 60637, USA

Fax: +1 7737026809

E-mail: michaelgluth@mac.com

Dr M B Gluth takes responsibility for the integrity of the content of the paper

Competing interests: None declared 